\title{
Heinrich Zangger und die Berufung Einsteins an die ETH. Sein Einfluss auf die Besetzung weiterer Physik-Lehrstühle in Zürich
}

Heinrich A. Medicus

\section{Summary}

Heinrich Zangger, 1874-1957, an eminent professor of forensic medicine at the University of Zurich, was a scientist with far ranging interests in many fields. In the course of his own research in physics, he became a close friend of Albert Einstein. When a chair in physics at his university or the Swiss Federal Institute of Technology in Zurich (ETH) was to be filled, Zangger was frequently actively involved in the search for a candidate. In particular, he engaged himself strongly, and in unconventional ways, in order to have Einstein appointed as professor at the ETH in 1912.

\section{Zusammenfassung}

Heinrich Zangger, 1874-1957, der bedeutende Gerichtsmediziner an der Universität Zürich, besass weitreichende wissenschaftliche Interessen auch ausserhalb seines Fachgebietes. Anlässlich einer physikalischen Arbeit kam er mit Albert Einstein zusammen, und die beiden wurden Freunde. Zangger nahm oftmals aktiven Anteil in Besetzungsfragen der Lehrstühle in Physik an den beiden Zürcher Hochschulen. Insbesondere setzte er sich kräftig und auf unkonventionelle Weise für die Berufung Einsteins 1912 an die Eidgenössische Technische Hochschule ein.

Prof. Dr. Heinrich A. Medicus, Department of Physics, Applied Physics and Astronomy, Rensselaer Polytechnic Institute, Troy, NY 12180, USA 


\section{Einleitung und biographischer Abriss}

Es ist ziemlich weit bekannt, dass der schon damals hoch angesehene theoretische Physiker Albert Einstein im Jahre 1912 durch die Vermittlung des Gerichtsmediziners Heinrich Zangger an die Eidgenössische Technische Hochschule (ETH) berufen wurde. (Bis 1911 hiess diese Eidgenössisches Polytechnikum, oftmals gekürzt «Poly».) Wenige wissen jedoch, wie stark und ausdauernd Zangger hiefür kämpfen musste, ferner, dass er sich schon vorher für Einsteins Berufung an die Universität verwandt hatte und auch in späteren Jahren bei der Besetzung von Lehrstühlen in Physik an den beiden Zürcher Hochschulen aktiv bei der Suche nach geeigneten Kandidaten mithalf. Um diesen uneigennützigen Einsatz zu verstehen, ist es nötig, näher auf die Persönlichkeit Zanggers einzugehen.

Heinrich Zangger ${ }^{1}$ wurde 1874 als einziges Kind eines recht gut gestellten Landwirtes in Bubikon im Zürcher Oberland geboren. Da er von eher zarter Konstitution war, aber schon frühzeitig durch seine aussergewöhnliche Intelligenz auffiel, verkaufte der Vater seinen Betrieb und zog nach Zürich, um dem Sohn den Besuch des Gymnasiums zu ermöglichen. Nach dem Medizinstudium in Zürich und Neapel verweilte Zangger zur weiteren Ausbildung in Paris. 1902 wurde er als a.o. Professor der Anatomie und speziellen Physiologie an die veterinär-medizinische Fakultät der Universität Zürich gewählt. 1906 übernahm er den Lehrstuhl für gerichtliche Medizin, welchen er bis zu seiner Emeritierung 1941 inne hatte. Mehrere äusserst attraktive Rufe, unter anderen die von Berlin und der Harvard University, konnten ihn nicht von Zürich weglocken. Nach langem Krankenlager starb Zangger 1957.

Zanggers oftmals spontane Hilfe zum Wohl seiner Mitwelt in Angelegenheiten, für welche er sich gar nicht hätte einsetzen müssen, sei hier an zwei Beispielen angeführt. 1906, während eines Pariser Studienaufenthaltes ereignete sich in Courrières in Nordfrankreich eine Grubenkatastrophe. Zangger fuhr dorthin, und durch sein Eingreifen wurden die schon eingestellten Rettungsarbeiten wieder aufgenommen. Noch über hundert Knappen konnten lebend geborgen werden, einer davon nach 23 Tagen!

Einmal, auf dem Weg in die Ferien, sah Zangger von der Bahn aus von einer Fabrik plötzlich gewaltige rotbraune Schwaden aufsteigen. Ihm war sofort klar, dass riesige Mengen von Nitrosegasen entwichen waren. Er unterbrach seine Fahrt, eilte zum Unglücksort und war einer der ersten Helfer. Mit

1 Für mehr über Zangger siehe Erinnerungen an Heinrich Zangger, hrsg. von H. Fischer, mit Beiträgen von Kollegen und Freunden und einem autobiographischen Aufsatz (Zürich, Orell Füssli Verlag) 1967. 
andern Ärzten nahm er sich zur Verhütung von Lungenödemen die ganze Nacht und den folgenden Tag der Opfer an; dann erst setzte er seine Reise fort.

Bei fast jeder grossen Katastrophe in Europa wurde Zangger als Experte beigezogen. Er war der eigentliche Begründer der Katastrophenmedizin.

Zanggers ausgedehntes wissenschaftliches Arbeitsfeld umspannte neben Gerichtsmedizin u.a. die Verhütung von Berufskrankheiten, rechtliche Probleme wie den Schutz von Minderjährigen und das Arztgeheimnis, sowie die Chemie der Kolloide. Er war in der Schweiz ein erfolgreicher Kämpfer gegen Bleizusätze zum Benzin und für die Eliminierung von Blei in Farben für Hausanstriche. 1932 wurde er in das Internationale Komitee vom Roten Kreuz gewählt, welchem er als aktives Mitglied bis 1947, später als Ehrenmitglied, angehörte.

Einstein charakterisierte Zangger in einem Brief vom 20. August 1952 an Carl Seelig folgendermassen:

«Sein Interessenkreis war sozusagen unbeschränkt, und er hatte auch Personen und Sachen gegenüber ein gutes Urteil, wo seine Fachkenntnisse eigentlich viel zu dünn waren. [...] Er sah die Sachen klar und scharf»².

Ähnlich urteilte auch Aurel Stodola, ein weltbekannter Professor für Wärmekraftmaschinen an der ETH:

«Dass sein [Zanggers] Interesse [in Physik] kein bloss informatorisch passives war, bewies er alsbald durch seine bekannte experimentelle Neubestimmung der Loschmidtschen Zahl [= Avogadroschen Zahl]. Aber auch in allen physikalischen Kolloquien um die Zeit, als die Theorien der Physik durch Schrödinger in ihren Fundamenten erschüttert und auf überwältigend grossartige Weise neu aufgebaut wurden, fehlte der liebe Kollege nicht $»^{3}$.

Als Professor besuchte er die Vorlesungen von Einstein und Debye. Gelegentlich vertrat er seine Kollegen in Prüfungen der Medizinstudenten in Pharmakologie, Pathologie und Physiologie. Während etlicher Jahre praktizierte er Lungenmedizin ${ }^{4}$.

\section{Zanggers Freundschaft mit Einstein}

Zanggers Tätgkeit auf dem Gebiet der Kolloide führte ihn mit Einstein zusammen. Er entwickelte eine Methode zur Bestimmung der Avogadroschen Zahl, die auf der Beobachtung der Brownschen Bewegung von Kolloiden be-

2 C. Seelig, Albert Einstein: Eine dokumentarische Biographie, Zürich (Europa Verlag) 1954, $129 \mathrm{f}$.

3 A. Stodola, Verantwortung aus Einsicht, in Festschrift Heinrich Zangger, Band 2, Zürich (Rascher Verlag) 1935, 971ff.

4 Persönliche Mitteilung von Prof. Zanggers Tochter, Gina Zangger. 
ruhte. Für die theoretische Erfassung des Problems benötigte er mathematische Hilfe. Er wandte sich um Rat an den bereits erwähnten Aurel Stodola, der ähnlich wie Zangger weitreichende Interessen in Physik und Geisteswissenschaften hatte. Dieser verwies ihn an Einstein, der damals Beamter am Patentamt in Bern war. Aus diesem Zusammentreffen entwickelte sich eine lebenslängliche Freundschaft.

Einstein sorgte später dafür, dass Zangger seinen Freund und ehemaligen Kollegen vom Patentamt, Michele Besso, kennen lernte. Die dauernde Freundschaft zwischen diesen drei ausserordentlichen Männern ist an anderer Stelle beschrieben worden 5 .

In den vierziger oder fünfziger Jahren plante Besso eine Biographie über Einstein. Zangger lieferte ihm hierfür ein paar flüchtig handgeschriebene Blätter mit äusserst aufschlussreichen stichwortartigen Notizen. Diese Blätter sowie seine Briefe an Besso wurden später an seine Tochter, Gina Zangger, zurückgegeben und wurden von mir bei der Arbeit für den oben erwähnten Artikel aufgefunden. Zu Anfang vermerkte Zangger, dass er sich 1905/06 zuerst wegen der mathematischen Behandlung der Brownschen Bewegung an Stodola wandte.

«Stodola sagte mir, dass er über meine Zählung der Brown[schen] M[olekular] Bewegung nichts Bestimmtes sagen könne. (1905/06 - vor meiner Heirat 1906 Stadthausquai [wo Zangger damals wohnte].) Ich solle zu Einstein nach Bern -.»

Einstein hatte damals schon einige Arbeiten in Wärmelehre, insbesondere einen Artikel «Allgemeine molekulare Theorie der Wärme» ${ }^{6}$ veröffentlicht, welcher gewisse Berührungspunkte mit Zanggers Problem hatte. Später, 1905, reichte Einstein nicht nur seine Dissertation an der Universität ein, sondern publizierte auch in den Annalen der Physik seine berühmte Arbeit über die Brownsche Bewegung ${ }^{7}$. Diese enthält am Schluss eine Formel, die für Zanggers Methode mit Modifikationen dienlich war. Da das Heft mit dieser Arbeit am 18. Juli 1905 ausgegeben wurde, ist es durchaus möglich, dass Stodola davon Kenntnis hatte. Eine andere Vermutung ist, dass Zangger Einstein schon einige Monate früher aufsuchte, da Einstein in einem Brief vom Mai an seinen Freund Habicht ${ }^{8}$ erwähnte, dass die

5 H. A. Medicus, The Friendship among Three Singular Men: Einstein and his Swiss Friends Besso and Zangger, Isis 85, 456ff., 1994.

6 A. Einstein, Allgemeine molekulare Theorie der Wärme, Ann. der Phys. 14, $354 \mathrm{ff} ., 1904$.

7 A. Einstein, Die von der molekularkinetischen Theorie der Wärme geforderte Bewegung von in ruhenden Flüssigkeiten suspendierten Teilchen, Ann. der Phys. 17, 549ff., 1905.

8 A. Einstein, The Collected Papers Gesammelte Schriften, Bd. 5, Die Schweizer Jahre, hrsg. von M. J. Klein, A. J. Knox, und R. Schulmann, (Princeton University Press) 1993, (im folgenden zitiert als Collected Papers 5) 31f., Dokument 27. 
«Bewegungen kleiner suspendierter Körper in der Tat beobachtet worden [sei] von den Physiologen [!], welche Bewegung von ihnen «Brown-sche Molekularbewegung〉 genannt wird.»

$\mathrm{Zu}$ dieser Zeit war Zanggers Lehrauftrag an der veterinär-medizinischen $\mathrm{Fa}-$ kultät umschrieben als «Vergleichende Physiologie, Chemie der Zelle, spezielle Physiologie und Pathologie der Milch». Es scheint somit, dass er nicht zuerst Hilfe suchte bei seinem Kollegen Alfred Kleiner, dem keineswegs hervorragenden Ordinarius der Physik an der Universität (und der ihn sicherlich auch an Einstein verwiesen hätte) sondern bei Stodola, dem Dampfturbinenbauer am Polytechnikum.

\section{Einsteins Ruf an die Universität Zürich}

In den erwähnten Notizen Zanggers für Besso steht weiter:

«Kleiner war erfreut. Ich musste zu Locher (der nicht hören wollte v[on] Einstein). Das zweite Mal kam ich mit einem grossen Triumpf, er wirkte».

Schon seit 1901 hatte Kleiner erfolglos beantragt, einen zweiten Lehrstuhl für Physik einzurichten. Zanggers Hilfe war ihm deshalb wertvoll. Albert Locher war damals als Regierungsrat Direktor des Erziehungswesens des Kantons Zürich. Allerdings existierte ausser Einstein noch ein anderer Anwärter auf einen solchen Lehrstuhl, Alfred Adler, Kleiners Assistent und Privatdozent für theoretische Physik, der auch Befürworter hatte?

Welchen von den beiden Kandidaten Kleiner vor Zanggers Besuch bevorzugt hätte, ist ungewiss ${ }^{10} .1901$ hatte Kleiner Einsteins Erwerbung des Doktordiploms (das Polytechnikum erhielt das Recht zur Doktorpromotion erst 1908) torpediert, da die eingereichte Arbeit über kinetische Gastheorie scharfe Kritik an den Gedankengängen des Wiener Theoretikers Ludwig Boltzmann enthielt. Dies war Kleiner aus kollegialen Gründen untragbar, so dass Einstein sein Begehren um Promovierung zurückziehen musste ${ }^{11}$. Anderseits schien Kleiner doch eine gute Meinung von Einstein zu haben, denn

9 Adler war der Sohn eines prominenten österreichischen Sozialdemokraten und selbst als solcher bekannt. 1917 schrieb Einstein in einem Brief an seinen Freund Besso (A. Einstein et M. Besso Correspondance 1903-1955, hrsg. von P. Speziali [Paris, Herman] 1972, Brief 31, 105f.), dass der Nachfolger Lochers, der sozialdemokratische Regierungsrat Heinrich Ernst, ihm später erzählte, dass der mehrheitlich sozialdemokratische Erziehungsrat einen Parteigenossen vorgezogen hätte, Adler ihm aber erklärt habe, dass Einstein der viel bessere Physiker sei und dass somit dieser gewählt werden solle.

10 Über die Anstrengungen Kleiners, Einstein an die Universität zu bringen, siehe auch R. Schulmann, Einstein at the Patent Office: Exile, Salvation, or Tactical Retreat? Science in Context 6, $17 \mathrm{ff} ., 1993$.

11 A. Reiser, Albert Einstein: A Biographical Portrait, with a foreword by Albert Einstein (London, Thornton Butterworth) 1931, 71 . 
um diese Zeit schrieb Einstein an Mileva Marić, seine zukünftige Frau, dass Kleiner ihm gesagt habe, er könne sich für eine Empfehlung auf ihn berufen. Kleiner habe auch seine Gedanken über das, was er später zur speziellen Relativitätstheorie entwickelte, interessant und publizierenswert gefunden ${ }^{12}$.

Anfangs 1903 teilte Einstein seinem Freund Besso in einem Brief ${ }^{13}$ von Bern aus mit, dass er es aufgegeben habe, den Doktor zu machen, «da mir das doch wenig hilft und die ganze Komödie mir langweilig geworden ist». Er wolle sich jedoch habilitieren. Einstein änderte aber seine Meinung, und im April 1905 reichte er an der Universität Zürich seine Dissertation über «Eine neue Methode zur Bestimmung der Moleküldimensionen» ein. Sie wurde von Kleiner und dem Mathematiker Heinrich Burkhardt als Korreferenten begutachtet, da nach Kleiners Ansicht «die Hauptleistung der Arbeit in der Behandlung von Differentialgleichungen besteht, also mathematischer Natur» sei ${ }^{14}$.

Nachdem 1907 Einsteins Habilitationsgesuch in Bern aus formalen Gründen abgewiesen worden war, ermutigte Kleiner ihn im nächsten Jahr, - folglich nachdem Zangger mit Kleiner gesprochen hatte - sich zu habilitieren. Er bot seine Hilfe an, da er ihn gerne auf dem zweiten, noch zu errichtenden Lehrstuhl gesehen hätte ${ }^{15}$.

Zangger erwähnte 1955 während eines Interviews mit Carl Seelig ${ }^{16}$, dass er 1908 als Dekan der medizinischen Fakultät Gelegenheit gehabt habe, sich für die Berufung Einsteins einzusetzen. Auf das Wintersemester 1909/10 wurde dieser zum Extraordinarius ernannt. Kleiner bemerkte später mit gewissem Recht, dass seine grösste Tat in der Physik die Begutachtung von Einsteins Dissertation und dessen Berufung an die Universität gewesen sei ${ }^{17}$.

\section{Einsteins Berufung an die Eidgenössische Technische Hochschule}

Einstein blieb nur drei Semester an der Universität Zürich, da er als ordentlicher Professor an die deutsche Universität Prag berufen wurde. Er hatte insofern Glück, als der Unterrichtsminister zunächst einen Österreicher vor-

12 Brief an Mileva Marić vom 19. Dezember 1901, Collected Papers 1 Dokument 130, 328.

13 Collected Papers 5, Dokument 5, $10 \mathrm{f}$.

14 Expert Opinion by Alfred Kleiner and Heinrich Burkhardt on Einstein's Dissertation, in Collected Papers 5, Dokument 31,35f.

15 A. Kleiner, Brief vom 8. Feb. 08 an Einstein, in Collected Papers 5, Dokument 80, 95 f.

16 C. Seelig, Helle Zeit - Dunkle Zeit (Zürich, Europa Verlag, 1956) 42.

17 G. Rasche und H. H. Staub, Physik und Physiker an der Universität Zürich 1833-1948, Vierteljahrsschrift der Naturf. Ges. in Zürich 124, 1979, 205ff., auf S. 211. 
zog, der aber den Ruf ablehnte, so dass Einstein, welchen die Prager Fakultät primo loco vorgeschlagen hatte, gewählt wurde ${ }^{18}$.

Der hauptsächliche Grund, die Zürcher Universität zu verlassen, war für Einstein die sehr kümmerliche Honorierung. Später, nach seinem Weggang von Prag, sagte er dies einem Wiener Journalisten und erklärte in einem Zeitungsartikel für die Neue Freie Presse ${ }^{19}$, dass Zürich den Vorteil seiner Lage an einem See und der Nähe der Berge habe. Er habe vor seinem Weggang von dort versprochen, unter annehmbaren Verhältnissen gern wieder zurückzukehren. Als sein Nachfolger in Zürich, Peter Debye, ebenfalls nach kurzer Zeit auf einen besser bezahlten Lehrstuhl in Holland wechselte, bemerkte Einstein in einem Brief vom 15. Nov. $1911^{20}$ an Zangger:

«Es ist fatal und tut mir aufrichtig leid, dass die Universität Debye schon wieder verlieren muss. Ein Ersatz dürfte schwer für ihn zu finden sein. Und dann besteht eben immer die prinzipielle Sache. Ein tüchtiger Kerl wird unbedingt bald wieder fortgehen von einer so bescheiden bezahlten Stelle.»

Obwohl Einstein nicht ungern in Prag war, blieb er auch hier nur drei Semester und kehrte nach Zürich zurück, jedoch an die ETH und als Ordinarius. Die bereits erwähnten Notizen Zanggers an Besso über sein Mitwirken an der Rückkehr sind aufschlussreich:

\footnotetext{
«Bundesrat Forrer spricht wegen Einstein mit mir. Ich zeigte ihm die Resultate, Brownsche Bewegung, Photoeffekt. Forrer und der Bundesrat will Einstein dem Poly zum Geschenk machen. also keine Berufung, keine Sitzung der Professoren. Reisen Sie nach Prag ... Grosse Wut am Poly - viele Poly Professoren - auf mich: Das geht Zangger einen Dreck an. Folgen der Angriffe der Polyprofessoren: Die Studenten nehmen sich meiner an: Es entstand ein Plakat: Hinter dem Poly hockt ein dünner Mann mit Schnauz [offensichtlich Zangger] - zieht am Seil, das zum Bahnhof geht, an dem Seil ist «Ein Stein` ein grosser Stein gebunden, zum Poly. Der Humor macht alles gut. Manche hatten keinen Humor. Die zwei Kollegen konnten meine 〈Untat nie vergessen bis ihnen etwas passierte.»
}

Diese etwas kryptischen Bemerkungen seien im folgenden näher erläutert.

Die Initiative bei Einsteins Rückkehr nach Zürich kann sehr wohl von Zangger ausgegangen sein, sicherlich im Einvernehmen mit Einsteins Studienfreund Grossmann, der nun Mathematikprofessor und zur Zeit Vorstand der Abteilung für Fachlehrer in Mathematik und Physik an der ETH war. Einstein schrieb später, am 22. September $1911^{21}$ aus Prag an Willem Julius in Utrecht, der ihn angefragt hatte, ob er an einem Ruf an diese Universität Interesse habe, er habe vor seinem Weggang von Zürich privatim versprochen, dort Mitteilung zu machen, bevor er eine andere Berufung annehme,

18 A. Kleinert, Anton Lampa und Albert Einstein: Die Neubesetzung der physikalischen Lehrstühle an der deutschen Universität Prag 1909 und 1910, Gesnerus 32, 284ff., 1975.

19 A. Einstein, Professor Einsteins Abgang von Prag, Neue Freie Presse, Nr. 17223, 5. 8. 1912, S. 8, nachgedruckt in Collected Papers 5, Dokument 414,499f.

20 Collected Papers 5, Dokument 305, 349.

21 Ebd., Dokument 288, 327. 
um der Leitung des Polytechnikums Gelegenheit zu geben, ihm auch einen Ruf zukommen zu lassen, falls sie es für gut finde. Zwar ist aus dieser Zeit kein Brief Einsteins mit einer solchen Mitteilung an einen seiner Zürcher Freunde bekannt. Es ist aber anzunehmen, dass Zangger bei seinem ersten Besuch in Prag im Frühling 1911, der mindestens teilweise wegen der Theorie zur Bestimmung der Avogadroschen Zahl unternommen war, auch eine eventuelle Rückkehr nach Zürich besprach, ferner dass vor einem zweiten Besuch in Prag im September ein Briefwechsel stattfand, welcher Zangger zum Handeln anspornte.

Dieser zweite Besuch Zanggers geschah im Auftrag von Bundesrat Ludwig Forrer, dem Vorsteher des Post- und Eisenbahn-Departementes, den Zangger aufgesucht und über Einsteins wissenschaftliche Arbeiten, insbesondere die Theorie der Brownschen Bewegung und des Photoeffekts (für welche Einstein später den Nobelpreis erhielt) informiert hatte. Dabei hatte er ihm vorgeschlagen, dass Einstein für die ETH gewonnen werden sollte. Zangger kannte Forrer, der Rechtswissenschaften studiert hatte, von Winterthur kam und dort im Polizeidienst tätig gewesen war, persönlich. Die Hochschule unterstand allerdings dem Departement des Inneren unter Bundesrat Josef Schobinger.

Bis Einstein schliesslich den Ruf erhielt, musste Zangger noch tüchtig und mit Ausdauer kämpfen. Dazu kam noch, dass zur selben Zeit die Universität Utrecht Einstein ein verlockendes Angebot machte ${ }^{22}$. Der Präsident der ETH, (mit offiziellem Titel Präsident des Schweizerischen Schulrates) Robert Gnehm, der vormals Professor für technische Chemie war, hatte natürlich auch ein gewichtiges Wort mitzusprechen und stellte sich gegen Einsteins Berufung. Um diese Zeit waren Diskussionen über die Reorganisation des Unterrichtes an der Hochschule im Gange. Am 30. September 1911 legte $\mathrm{Gnehm}$ in einem langen Bericht ${ }^{23}$ an Bundesrat Schobinger seine Gründe dar:

«[...] Herr Einstein ist eine ausgesprochene Gelehrtennatur. Seine wissenschaftlichen Arbeiten zeugen nach Ansicht der Sachverständigen [...] von Originalität und Tiefe der Gedanken, von genialer schöpferischer Kraft. Dagegen ist er kein hervorragender Dozent und entbehrt einer ausgesprochenen Lehrbegabung. Für die Ausübung einer umfangreichen Lehrtätigkeit, wie sie mit einer Professur für allgemeine Physik oder technische Phyik an unserer Hochschule verbunden ist, wäre Herr Einstein nicht geschaffen. Das entspräche weder seiner Veranlagung noch seiner Neigung. Will man dem genialen Forscher gerecht werden, [...] müssten [wir] eine Professur für theoretische Physik schaffen mit einer ganz bescheidenen Lehrverpflichtung [...] für eine kleine Elite der Vorgerückteren und Begabteren an unserer VIII. Abteilung (Abt. für Fachlehrer in Mathematik und Physik) und vielleicht auch für diesen und jenen mathematisch besonders veranlagten Ingenieur. [...]

22 W. Julius, Ebd., Dokument 289, 329.

23 Schweiz. Bundesarchiv, Bern, E8 (B), Schachtel 90. 
Es unterliegt keinem Zweifel, dass durch die Gewinnung Einstein's [...] die Rangstellung unserer VIII. Abteilung eine wertvolle Stärkung erhielte. Ob wir im gegenwärtigen Moment einen solchen - man möchte fast sagen - Luxus (im besten Sinne) erlauben sollen, bedarf einer eingehenden Prüfung.

[...] es handelt sich um einen Physiker; da genügt nicht die blosse Anstellung und $\mathrm{Zu}$ weisung eines Auditoriums; es muss auch ein Laboratorium mit Zubehör (Assistenten etc.) zur Verfügung stehen. [...]

Schon seit einiger Zeit beschäftigen wir uns mit der Frage der Reorganisation des physikalischen, speziell des elektrotechnischen Unterrichtes, der einer Reform dringend bedarf ...

Der gesamte Physikunterricht (mit Ausschluss der praktischen Elektrotechnik, die durch die Professoren [Jean Lucien] Farny und [Walter] Wyssling vertreten ist) wird zur Zeit erteilt von 2 ordentlichen Professoren ([Friedrich] Weber und [Pierre] Weiss) und einem Titular-Professor ([Alfred] Schweitzer). Sehr wahrscheinlich wird man dazu gelangen, das Gebiet der theoretischen Elektrotechnik abzulösen und einer neuen Lehrkraft zu übertragen. Für dieses Gebiet könnte Herr Einstein nicht in Betracht fallen. Sollte er mit in das Reorganisationsprojekt einbezogen werden, so erhielten wir 4 ordentliche Professoren für Physik [Gnehm zählte offenbar den Elektrotechniker mit!] neben einem Titularprofessor. Abgesehen davon, dass die Laboratoriumsverteilung bei einem derartigen Personalbestande ein schwer zu lösendes Problem bildet, wird auch der Einfluss auf das Budget genau erwogen werden müssen. [...]

Zusammenfassend lässt sich unser Standpunkt etwa folgendermassen kennzeichnen: In der allernächsten Zeit wird es kaum möglich sein zu entscheiden, ob eine Berufung des Herrn Einstein versucht werden soll oder nicht. Die Frage ist so eng mit den vorstehend erwähnten Reorganisationsbestrebungen verknüpft, dass sie nur mit diesen erledigt werden kann $[\ldots]$

Durch diese Verzögerung laufen wir allerdings Gefahr, dass die Berufung nach Utrecht inzwischen perfekt wird. Bei der grossen Vorliebe, die Herr Einstein für Zürich haben soll, halten wir ihn nicht für verloren. In dem Moment, wenn wir ihm einen auf ihn zugeschnittenen Wirkungskreis anzubieten in die Lage kommen sollten, wird wahrscheinlich der Finanzpunkt eine Rolle spielen, an dem aber allfällige Unterhandlungen kaum scheitern dürften.»

Auf Forrers Weisung wurde Zangger über alle Schritte voll informiert; er sandte ihm eine Kopie von Gnehms Bericht und bat um seinen Kommentar. Zangger antwortete Forrer - in ab und zu nicht völlig literarischem Deutsch - am 9. Oktober ${ }^{24}$ :

«[...] I. Für eine Lehramtsstelle, wie die eidg. tech. Hochschule sie hat, ist ein ordentlicher theoret. Physiker eine Forderung - dies Bedürfnis hängt mit der Reorganisation, von der schon lange gesprochen wird, recht wenig zusammen, zumal

II. Einstein gar kein Laboratorium noch Assistenten braucht, sie weder hier noch in Prag noch in Utrecht hätte. Ich bin überzeugt, dass ihm Prof. Weiss, der die Bedeutung Einsteins kennt, sofort Wege finden würde durch sein Institut Einstein das Nötige zu beschaffen. [...] Ich selbst könnte ihm zur Not für einige Jahre Unterkunft bieten [...]

III. Die sehr gewichtige Supposition Einstein wolle eine Stelle mit wenig Lehrverpflichtung (ein auf ihn zugeschnittener Wirkungskreis) ist unbegründet, ich kann diese Annahme durch Einsteins eigene Worte widerlegen: Er sagte mir einmal «Ich lese gerne die gesammte theoretische Physik, ich werde mich doch danach richten, was für Bedürfnisse die Schule hat - So hat er es auch hier u in Prag gehalten (6 Stunden Vorlesungen plus einige Stunden Seminar u Übungen u Doctoranden)

IV. Einstein als Lehrer. Darüber erlaube ich mir mein eigenes Urteil, weil ich Einstein, so lange er in Zürich war, wöchentlich einige Stunden hörte.

24 H. Zangger an L. Forrer, in Collected Papers 5, Dokument 291, 331f. 
Er ist kein guter Lehrer für denkfaule Herrn, die nur ein Heft voll schreiben wollen u es auswendig lernen wollen für das Examen, es ist kein Schönredner, aber wer lernen will ehrlich, tief innerlich seine physikal. Gedanken aufzubauen, alle Praemissen umsichtig zu prüfen die Klippen u Probleme zu sehen, die Zuverlässigkeitsgrenzen einer Überlegung zu übersehen, der findet in Einstein einen erstklassigen Lehrer, denn alles das kommt im Vortrag zum suggestiven Ausdruck, der zum Mitdenken zwingt u die Weite des Problems aufrollt; sein Vortrag hat grosse Ähnlichkeit mit dem Vortrag von [Marie] Curie u [Paul] Langevin, die ich, wie Einstein je ein Jahr [in Paris] hörte.»

Zangger schloss diesen Brief an Bundesrat Forrer mit der Ankündigung, dass er mit Einstein zu ihm am 14. Oktober nach Bern käme. Einstein hielt sich damals gerade in Zürich auf für eine Reihe von Vorträgen in einem Ferienkurs für Mittelschullehrer. Forrer verzeichnete in seinem Notizbuch diesen Besuch sehr kurz ${ }^{25}$ : «Samstag Besuch von Prof. Zangger u. Einstein». Der am selben Tag getätigte Kauf von Steinpilzen für Fr. 6 auf dem Markt war wesentlich detaillierter!

\section{Am 22. Oktober erwähnte Einstein in einem Brief an Zangger:}

«Das von Forrer an Sie und von Ihnen an meine Frau gesandte Telegramm [...] hat mich unbedingt gefreut. [...] Die freundliche Gesinnung und das Vertrauen eines solchen Mannes wie Forrer zu haben, ist mir eine grosse Freude» ${ }^{26}$.

Forrer war demnach bereit, sich energisch für Einsteins Berufung einzusetzen, nachdem er ihn persönlich kennengelernt hatte.

Gnehm hatte nicht aufgegeben und versuchte in einem Schreiben vom 21. Oktober an Bundesrat Schobinger, dem Vorsteher des Departements des Innern, die Argumente von Zangger zu widerlegen ${ }^{23}$. Sein Schreiben legt klar dar, dass er nicht viel Ahnung davon hatte, was theoretische Physik ist:

«ad I: Theoretische Physik wird behandelt. Im letzten Jahr: Theorie der absoluten magnetischen und elektrischen Messungen (2 Std.); Einführung in die Wechselstromtheorie (2 Std.); Elektrische Schwingungen (2 Std.); Elektromechanik (2 Std.); Leçons expérimentales sur la radioactivité (1 Std.); Ionisation, Radioactivité (1 Std.)

ad II. Einem Physiker kein Laboratorium zuzusichern und ihn einfach auf das Wohlwollen eines Kollegen zu verweisen, hielte ich nicht für ein glückliches Argument.

ad III. Die Annahme, ich supponiere Herr Einstein wolle eine Stelle mit wenig Lehrverpflichtung entspricht nicht der Wirklichkeit ...

ad IV. «denkfaule Herren - kein Schönredner,» das spricht ja gänzlich für mein Urteil: Hr. Einstein ist ein Lehrer für Vorgerücktere und besonders Begabte, nicht für die grosse Masse der Studierenden, für die der Unterricht in allgemeiner und technischer Physik bestimmt ist.

Mit dieser Ansicht stimmen alle Herren überein, mit denen ich früher und in letzter Zeit über Herrn Einstein gesprochen habe; darunter sind mehrere Physiker und Mathematiker, die ihn, seine wissenschaftlichen Arbeiten und seine Tätigkeit an der Universität ziemlich gut kennen.

In der Wertschätzung des Herrn Einstein als Forscher stimme ich mit Herrn Prof. Zangger völlig überein; dessen Name würde unserer VIII. Abteilung erhöhten Glanz verleihen.»

25 Forrers Notizbücher sind in der Handschriftenabteilung der Zentralbibliothek Zürich deponiert, Ms. Z II 740.

26 Collected Papers 5, Dokument 297,340f. 
Zanggers wiederholte Interventionen wurden dem Bundesrat schliesslich lästig. Schobinger schrieb daher am 9. November 1911 an Zangger ${ }^{23}$, indem er zunächst Zanggers Schriftstück anführte und dann auf Gnehms Replik Bezug nahm:

«Diese Ausführungen werden es Ihnen gerechtfertigt erscheinen lassen, dass die Bundesbehörden sich der Anschauungsweise des Herrn Schulratspräsidenten über die Opportunität einer sofortigen Gewinnung des Herrn Prof. Einstein unterordnen und sich weiteren Einwirkungen zu Gunsten des letzteren enthalten.»

Zangger muss seinem Freund in Prag über diesen Rückschlag informiert haben, denn am 15. November schrieb Einstein ${ }^{27}$ :

«Lieber Freund Zangger! Seien Sie nicht so wütend und temperamentvoll! Es thut mir leid, dass ich Sie in Ihrer Ruhe, die Sie doch sowieso nicht haben, durch diese öde Berufungsaffäre gestört habe. Ich denke einfach nicht mehr daran. Nach Utrecht habe ich soeben abgeschrieben, und die lieben Züricher können mich auch ... gern haben bis auf Sie. Ich bitte Sie nur das eine: beschäftigen Sie sich nicht mehr mit der Affäre.»

Inzwischen hatte sich die Situation in Bern sehr verändert. Schobinger erkrankte am 11. November, zwei Tage nach seinem Brief an Zangger, an einer Brustfellentzündung, an der er am 27. November starb. Vermutlich konnte infolge seines Ausfallens Forrer sich nunmehr bei seinen Kollegen erfolgreich für Einstein einsetzen. Auch in Zürich wurde man an der Hochschule rührig. Am 16. November muss Einstein von Grossmann einen Brief erhalten haben mit der Anfrage, ob er eine Lehrstelle für theoretische Physik am Polytechnikum annehmen würde. Er schrieb nämlich davon am selben Tage nach Utrecht ${ }^{28}$. Da er aber wusste, dass die Utrechter Stelle nun an Debye ginge, befürchtete er, dass man es in Zürich deshalb plötzlich nicht mehr so eilig habe mit seiner Berufung an die ETH. Er bitte deshalb die Utrechter, mit der offiziellen Anfrage an Debye noch ein wenig zu warten. Auch Debye musste ihm versprechen, in der Schweiz nichts von der Entwicklung der Lage verlauten zu lassen ${ }^{29}$. Grossmann antwortete er am 18. November in positivem Sinne ${ }^{30}$. Zwei Tage später telegrafierte er Zangger «Habe Grossmann zugesagt» ${ }^{31}$ und schrieb ihm ausserdem einen Brief ${ }^{32}$ :

«Sie sind durchgedrungen, unglaublicher Weise: Ich hätte sehen mögen, wie Sie das gemacht haben. [...] Nehmen Sie noch meinen innigsten Dank entgegen dafür, dass Sie sich meinetwegen in so prekäre Unternehmungen gestürzt haben.»

27 Ebd., Dokument 305, 349.

28 Einsteins Brief an W. Julius, Ebd., Dokument 306, 350f.

29 Brief von P. Debye vom 3.11.1912 an A. Sommerfeld, Deutsches Museum, München, Handschriften-Sammlung, 1977-28 (A,61/11).

30 Collected Papers 5, Dokument 307, 351.

31 Ebd., Dokument 309, 353.

32 Ebd., Dokument 308, 352f. 
Das Schulratsprotokoll vom 2. Dezember ${ }^{33}$ vermerkte, dass nach dem Rücktritt [Hermann] Minkowskis [des bedeutenden Mathematikers, der nach Göttingen berufen wurde] es «zu einer Verkümmerung des Stundenplans für die oberen Semester an der VIII. Abteilung [für Fachlehrer in Mathematik und Physik]» gekommen sei.

«Die bisherigen Versuche zu dessen Behebung scheiterten an der Personenfrage. Neuerdings wird von verschiedenen kompetenten Fachmännern auf Dr. A. Einstein, z.Z. Prof. für theor. Physik an der deutschen Universität Prag, als einen für unsere Zwecke geeigneten Gelehrten, hingewiesen. Der Schulrat, in der Absicht, für unsere Hochschule eine Kraft ersten Ranges auf dem Gebiet der modernen theoretischen Physik zu gewinnen, nach gewalteter Diskussion, beschliesst: Der Präsident wird ermächtigt, mit Prof. Dr. Einstein [...] in Unterhandlung zu treten und dem Schulrat über das Resultat Bericht und eventuell Antrag vorzulegen.»

Somit wurde der vakante Lehrstuhl den Mathematikern weggenommen und der Physik zugeteilt. Es ist sehr wohl möglich, dass erst nach dem Ausscheiden Schobingers und nach Einsteins Besuch bei Forrer diese Lösung aufkam und dass der Mathematiker Grossmann sie als erster vorschlug.

Falls Einsteins Stelle gleich von Anfang an als «Geschenk Forrers und des Bundesrates», d.h. als zusätzliche Professur ohne Belastung des Personalbudgets der ETH, aufgefasst worden wäre, wie aus Zanggers Notizen herauszulesen wäre, ist schwer einzusehen warum Gnehm sich so lange ablehnend verhielt. Der Widerstand gewisser ETH-Professoren erklärt sich daraus, dass es manchem nicht gefallen hat, dass ein Mediziner der Universität unter Umgehung der regulären Berufungskanäle jemanden, so bedeutend er auch sei, der ETH aufzudrängen versuchte. Wer die Physiker waren, die sich negativ verhielten und auf die sich Gnehm bezog, ist nicht festzustellen. Sicherlich war nicht Pierre Weiss darunter, der bedeutende Erforscher des Magnetismus, der nebst andern ein positives Gutachten über Einstein abgegeben hatte. Es könnte aber Friedrich Weber gewesen sein, der Einstein nach dessen Diplom an der ETH nicht als Assistenten angestellt hatte, sondern zwei Ingenieure vorzog. Einsteins früherer Kollege an der Universität, Kleiner, hat sich vielleicht auch ungünstig über ihn ausgesprochen. Erstens war er nicht begeistert, dass Einstein ihn schon nach drei Semestern verlassen hatte, nachdem ihm kurz vorher eine Erhöhung seines Gehaltes zugesprochen worden war und er versprochen hatte, nicht von Zürich wegzugehen. Zweitens hatte Einstein während seiner Zeit an der Universität Meinungsverschiedenheiten mit Kleiner betreffend die Besetzung eines Lehrstuhls inMathematik ${ }^{34}$. Gnehm meinte sicher Ernst Meissner, den Professor für Me-

33 Protokoll des Schweiz. Schulrates Nr. 117, Schulratsarchiv ETH.

34 Collected Papers 5, Dokument 369, Fussnote 6, 428. 
chanik, der später - Zanggers Notiz «bis ihnen etwas passierte» scheint darauf hinzuweisen - infolge Nachlassens seiner geistigen Kräfte vorzeitig emeritiert werden musste. An der ETH lehrte in Physik ausserdem seit 1901 noch der Titularprofessor Alfred Schweitzer, der dann gleichzeitig mit Einstein auf das Wintersemester 1912 zum Professor ernannt wurde.

Für die Meinungsänderung im Bundesrat und von Gnehm war auch der Umstand wichtig, dass das Parlament gerade um diese Zeit den sehr grosszügigen Baukredit für den Ausbau der ETH beschloss, was in Zürich mit 22 Kanonenschüssen gefeiert wurde. Es hätte schlecht in dieses Bild gepasst, jetzt die Möglichkeit, eine erstklassige Kraft zu gewinnen, ungenützt vorbeigehen zu lassen.

Eine Woche nach der oben erwähnten Schulratssitzung informierte Gnehm Einstein, dass beabsichtigt sei, die nach dem Rücktritt Minkowskis vakant gebliebene Lehrkanzel wenn möglich auf Oktober 1912 wieder zu besetzen $^{35}$. «Wir suchen jemanden zu gewinnen, der qualifiziert wäre [...]» Er möge auch seine Ansichten über den geplanten Ausbau des Studienplanes für Physiker und Mathematiker an der ETH äussern, wenn möglich mündlich in Zürich, oder er, Gnehm, könne ihn nach Neujahr auch in Prag besuchen.

Nun erhielt Einstein am 10. Dezember von Hendrick A. Lorentz eine implizite Anfrage, zu ihm nach Leiden zu kommen ${ }^{36}$. Deshalb schrieb er gleichen Tages an Grossmann, er solle in Zürich veranlassen, dass die Verhandlungen «prestissimo» eingeleitet würden ${ }^{37}$. Lorentz erklärte er am 12. Dezember seinen Standpunkt ${ }^{38}$ :

«Was mich am meisten verpflichtet, eine Berufung nach Zürich anzunehmen, das ist der Umstand, dass sich ein dortiger Freund und Kollege [Zangger] mit meinem Wissen emsig bemüht hat, eine solche Berufung zu veranlassen, worauf er gestützt auf frühere Äusserungen von mir fest darauf zählte, dass ich eine Berufung annehmen würde. Er schrieb mir kürzlich, dass es äusserst peinlich, ja sogar verletzend für ihn wäre, wenn eine Absage von mir erfolgte.»

Grossmann, der Gnehm über den Ruf aus Utrecht informiert hatte, schlug Einstein vor, zur Beschleunigung der Berufung nach Zürich zu kommen. Etwas widerwillig schrieb Einstein am 13. Dezember an $\mathrm{Gnehm}^{39}$, dass er ungern zu einer Besprechung nach Zürich reise, da dies einen Zeitverlust von drei Tagen bedeute. Er werde es aber, wenn wirklich nötig, tun. Etwa gleich-

35 Brief vom 8. Dez. 1911, Ebd., Dokument 317, 365.

36 Brief vom 8. Dezember 1911, Ebd., Dokument 318, 366.

37 Ebd., Dokument 319, $366 f$.

38 Ebd., Dokument 320, 367f.

39 Ebd., Dokument 324, 371. 
zeitig informierte er auch Zangger ${ }^{40}$ : «Damit Sie sehen, wie Ihre Saat wenn auch nicht spriesst, so doch keimt, schicke ich Ihnen Gnehms bauernschlauen Brief nebst meiner Antwort dazu.»

Trotz seiner Brüskierung durch den Brief von Schobinger war Zangger immer noch aktiv. Am 14. Dezember schrieb er Gnehm ${ }^{41}$ :

«Wie sich die Frage mit Einstein entwickelt hat, weiss ich nicht, aber zufällig vernahm ich von Wien, dass man ihn zu einem Vortrag nach Wien einladen wird auf Ende Januar mit dem Gedanken eine Stellung zu schaffen etc. Ich möchte es auch diesmal nicht auf dem Gewissen haben diese Gefahr nicht bei Zeit vermerkt zu haben.»

Am 16. Dezember telegrafierte Gnehm an Einstein, nach Zürich zu kommen $^{42}$. Die beiden trafen an 21. Dezember zusammen. An Weihnachten schrieb Einstein an Zangger:

«Ich bin glücklich über meine Züricher Erlebnisse und danke Ihnen nochmals herzlich dafür, dass Sie für diese Sache Ihre Persönlichkeit mit solcher Intensität einsetzten. [...] Einstweilen freue ich mich sehr darauf, dass wir zusammen wieder regelmässig verkehren und fachsimpeln können» ${ }^{43}$.

Es ist unklar, wie Studenten vom Einsatz Zanggers erfuhren und für welchen Anlass das in Zanggers Notizen erwähnte Plakat erstellt wurde mit seiner Karikatur, «Ein Stein» an einem Seil vom Hauptbahnhof zum Poly heraufziehend $^{44}$.

Am 23. Januar 1912 informierte Gnehm Einstein, dass der Schulrat dem Bundesrat seine Wahl vorgeschlagen habe und dass die Angelegenheit in etwa 14 Tagen formell erledigt werde ${ }^{45}$. Eine Woche später schrieb Zangger an Einstein, dass er an Forrer, der jetzt Bundespräsident war, geschrieben habe, man möge Einstein, der andere Anfragen habe, schnell das definitivum zukommen lassen.

«Forrer freute sich sehr das beweist, dass er mir telegraphiert $11^{\mathrm{h}}$ die Wahl, $11^{20}$ Bericht. Die Schweizer sind doch dabei - die dabei sind. Mein Telegramm, das ich sofort aufgab, haben Sie hoffentlich bekommen ${ }^{46}$.

40 Ebd., Dokument 325,371f.

41 ETH Schulratsarchiv, Aktennummer 1349.

42 Collected Papers 5, Dokument 326, 372.

43 Ebd., Dokument 330, $378 \mathrm{f}$.

44 Auf eine Anregung der ETH-Leitung organisierte die Studentenschaft Mitte Januar einen Fackelzug als Ausdruck des Dankes an das Parlament für den grosszügigen Ausbau der Hochschule. Das Plakat kann vielleicht gebraucht worden sein für den Aufruf zu dieser Demonstration, oder sogar im Umzug selber, in welchem auch mehrere Musikkorps marschierten, die Ungarn historische Motive stilisierende Kappen trugen und die Italiener die Trikolore mitführten (Züricher Post, 18. Januar 1912). Denkbar ist allerdings auch, dass das Plakat aus Anlass des Bezuges des Neubaus für die gerichtlich-medizinische Institut im Frühjahr 1912 entstanden ist.

45 Collected Papers 5, Dokument 341, 392.

46 Brief vom 30. Januar 1912, Ebd., Dokument 347, 397f. 
So endete schliesslich das zähe Ringen Zanggers mit seinem Sieg. Am 2. Februar dankte Einstein dem Bundespräsidenten Forrer «für die rasche Ernennung ans Polytechnikum $»^{47}$.

Zur grossen Enttäuschung Zanggers und der ETH blieb Einstein nur drei Semester in Zürich. Im Sommer 1913 reisten Max Planck und Walther Nernst nach Zürich, um bei Einstein zu sondieren, ob er mit einem grosszügigen Angebot an die Preussische Akademie in Berlin gelockt werden könnte. Sie hatten damit Erfolg, und im Frühjahr 1914 verliess Einstein Zürich.

\section{Zanggers dritte Aktion zur Rückgewinnung Einsteins}

Mitte 1918, als die Lebensverhältnisse in Deutschland recht schwierig wurden, machte Zangger erneut einen Versuch, Einstein für Zürich zurückzugewinnen. Zusammen mit Edgar Meyer, dem Experimentalphysiker an der Universität, plante er für ihn eine Doppelprofessur an der ETH und der Universität, wie solche zwischen den benachbarten Hochschulen in andern Fächern schon bestanden.

Auf eine Redewendung in einem Brief Einsteins anspielend, schrieb ihm Zangger ${ }^{48}$ :

«Lieber Freund Einstein: Wie kommen Sie dazu, sich mit 40 Jahren eine Ruine zu nennen, wie ein Ei, nachdem die Brütung erledigt. Sie haben instinctives Bedürfnis nach Luft - wollen diesen Herbst kommen. Also: RegRat [Erziehungsdirektor Heinrich] Mousson hat mit 〈Ihrer Excellenz [Ministerialdirektor im Kultusministerium Otto] Naumann〉 gesprochen und will mit dem Schulratspraesident [der ETH] sprechen - ich gehe zum Bundesrat ${ }^{49}$. (Man glaubt mir sogar sehr viel, weil ich ausser Einstein auch für Meyer für Debeye sprach u ich ausserhalb stehe) Sie stehen also für sich vor einer klaren Situation: Entweder frische gesunde Luft - einige Vorlesungen - wenn Sie gesund sind - zusammen für Poly-Universität u mit Freuden aufgenommen oder das ungesund u bequemere mit dem ruinösen Gefühl.»

Am 16. August 1918 antwortete Einstein ${ }^{50}: \ll$ Sie sind ein Plaggeist von rührender Güte ...», er wollte aber nicht von Berlin fort.

"Andererseits betrachte ich nach wie vor Zürich als meine wirkliche Heimat und die Schweiz als das Land, dem allein ich mit meiner Neigung zugethan bin ... Ich will in Zürich öfters Vortragszyklen abhalten von etwa 6 Wochen; etwa zweimal im Jahre. [...] Wenn Sie meinen, können wir Ende September gleich anfangen oder Februar. Ich verlange dafür nicht mehr als die Vergütung meiner Auslagen. [...] es würde mir sehr grosse Freude machen, den Plan durchzuführen.»

47 Ebd., Dokument 351, 402.

48 Zangger an Einstein, vor 12. August 1918, Collected Papers 8 (in Vorbereitung).

49 Da Bundesrat Forrer nicht mehr im Amte war, wird Zangger entweder an Gustave Ador aus Genf, der dem Departement des Innern vorstand, gedacht haben, oder, noch wahrscheinlicher, an den Zürcher Bundesrat Robert Haab, der bis vor kurzem Gesandter in Berlin gewesen war und dort wohl Einstein kennen lernte.

50 Einstein an Zangger, 16. August 1918, Collected Papers 8. 
Zwei Tage später informierte Einstein auch seinen Freund Besso über seinen Gegenvorschlag, diesen sogar etwas mehr präzisierend, «zweimal im Jahr für je 4-6 Wochen nach Zürich und halte jedesmal einen Vortragszyklus von etwa 12 Vorlesungen ${ }^{51}$.

Die Mühlen in Zurich mahlten aber nicht so schnell. Es mussten noch einige administrative Details abgeklärt werden. Am 3. Oktober 1918 schrieb Besso, der von Zangger auch in die Sache eingespannt war, an Zangger ${ }^{52}$ :

«Edgar M[eyer] frägt an, ob die Sache von der Universität allein (ohne Poly) betrieben wird (in welchem Falle ihm die Eitelkeit helfend zustatten käme).... Dabei tauchte aber vor mir die Aufgabe auf, zu vermeiden, dass diese Eitelkeit wieder umgekehrt zu einem Reibungsanlass zwischen Poly und Uni werde: In diesem Sinne wäre es (vielleicht ?!?) gut, Sie hätten bald Gelegenheit, Gnehm und [ETH Rektor Emil] Bosshard von der Sache zu erzählen.»

Anfangs 1919 kam Einstein nach Zürich und hielt an der Universität den Vorlesungszyklus.

Da Einstein versprochen hatte, weitere solche Zyklen zu geben, fragte ihn Zangger am 22. Oktober 1919,,$^{53}$ :

«[...] Was macht Ihr Lehraufrag hier? Ich höre von Prof. Meyer, dass Sie nicht mehr kommen wollen. [...] Schreiben Sie mir auch wegen Lehrauftrag. Vom krummen Licht habe ich schon der Behörde vor Jahren den Kopf vollgemacht. Galilei, Newton, Einstein proklamiert - also wenn Sie den Lehrauftrag wollen, resp. behalten, wäre das allen eine Freude.»

Kurz vorher war nämlich während einer Sonnenfinsternis die Beugung des Lichtes im Schwerefeld der Sonne bestätigt worden, die Einsteins allgemeine Relativitätstheorie vorausgesagt hatte. Am Weihnachtsabend 1919 schrieb Einstein an Zangger ${ }^{54}$ :

[...] Ich lese nicht mehr an der Züricher Universität. Die Physik ist jetzt in Zürich so gut vertreten, dass es Überhebung wäre, und es fiele mir auch schwer, so viel Zeit zu erübrigen. [...]

Ein zusätzlicher Grund mag sein, dass Einsteins Erfahrungen in Zürich nicht ganz ungetrübt waren, denn in einem undatierten Brief an Zangger schrieb er später, $1921^{55}$ :

«[...] Und offen gestanden finde ich die Schweizer besonders kleinlich. Ich werde nie vergessen, wie mir der Rektor [der Universität, Theodor] Vetter bei meinen Relativitätsvorträgen, die ich vor ein paar Jahren in Zürich hielt, vorwarf, dass die Heizung meines Hörsaales so viel Geld kostete! Das würde kein Franzose fertig bringen. Weiss Gott, wo Sie Ihr Blut herhaben, dass Sie so ganz anders eingestellt sind, als der Nordschweizer im allgemeinen ist, dessen Gehirn sich ständig um die Fränkli herumdreht. [...]»

51 Einstein an Besso vom 20. August 1918, Correspondance Brief 46, $132 \mathrm{ff}$.

52 Im Nachlass Zangger.

53 Zangger an Einstein, 22.10.19, Collected Papers 10 (in Vorbereitung). Alle Zitate aus dem Briefwechsel Zangger-Einstein ab 1919 sind den Abschriften von Helen Dukas in den Wissenschaftshistorischen Sammlungen der ETH Bibliothek entnommen, im folgenden als ETH zitiert.

54 Ebd.

55 Einstein an Zangger, 1921, ETH und Collected Papers 10. 
In Leiden hatte Kammerlingh Omnes jedoch mehr Glück mit seiner Offerte an Einstein für eine dreijährige Gastprofessur mit nur ein paar Wochen Lehrverpflichtung, da Einstein diese annahm. Am 9.April 1920 schrieb Zangger enttäuscht an Besso ${ }^{56}$ :

«Die beiliegende Karte habe ich von Einstein erhalten. Ich werde daraus nicht klar, ob es ihm gut geht, warum er eher nach Holland geht, als in die Schweiz.»

\section{Zanggers weitere Bemühungen um die Besetzung physikalischer Lehrstühle in Zürich}

Zangger betätigte sich weiterhin recht aktiv, wenn ein Lehrstuhl der Physik an einer der Hochschulen zu besetzen war, und er zählte dabei auf den Rat Einsteins. 1919 wurde Pierre Weiss an der ETH, der durch seine Forschung über Magnetismus sehr bekannt war, an die Universität Strasbourg berufen. Im oben erwähnten Brief vom 22. Oktober schrieb Zangger an Einstein:

«Debye war hier! Gnehm sagte mir, [nachdem er ihn in St. Moritz getroffen hatte] er sei überzeugt, dass Debye der beste sei. Zuerst stehen heute [Jean] Perrin - [Auguste] Piccard [seit 1915 Privatdozent an der ETH] in Frage. [...] Im Moment weiss ich noch nicht, wie ich es anstellen kann, die Menschen zu packen. Ich bin zur Zeit etwas geknickt, weil man sich wieder ziemlich bemüht, mich auszuschalten - ich stand an zu exponiertem Posten - war zu oft gewalttätig für das Rechte - das hasst man.»

Vermutlich im Januar 1920 benachrichtigte Einstein Zangger ${ }^{57}:$ «Vor einigen Tagen sah ich Debye. Man bemüht sich hier [in Göttingen] krampfhaft ihn festzuhalten, in Zürich ebenso, ihn zu gewinnen.».

Zangger hatte offensichtlich Einstein berichtet, dass die ETH in erster Linie Schweizer berufen wolle und hatte ihm einige Namen zur Beurteilung vorgelegt. Einstein anwortete auf einer Postkarte an Zangger am 20. März ${ }^{58}$ :

«... Den Gesichtspunkt, Schweizer anstellen zu wollen, begreife ich durchaus. [Paul] Scherrer ist fähig und tüchtig, wenn auch kein eigentlicher Theoretiker. [August] Bernoulli aber ist unfähig, seine Ernennung wäre Sabotage. H. A. Lorentz und Ehrenfest bieten [Paul] Epstein eine bezahlte Stellung in Leiden an, trotzdem dort nichts frei ist. Die Holländer tun viel für Wissenschaft um ihrer selbst willen. Daran fehlt es noch in der Schweiz. Man sollte nicht vergessen, dass Reichtum verpflichtet.»

Debye erhielt die Berufung an die ETH und nahm seinen Mitarbeiter Paul Scherrer mit, der, wie auch Piccard, zum Professor ernannt wurde. 1922 schrieb Zangger an Einstein ${ }^{59}$ :

56 Im Nachlass Zangger.

57 Einstein an Zangger, Jan. 1920, Ebd.

58 Postkarte von Einstein an Zangger, 20. März 1920, Ebd.

59 Zangger an Einstein, 1922, Ebd. 
«... Diesen Winter hörte ich das ganze Kolleg Debyes über Struktur der Materie - sehr gut, nur zu kurz, aber viele Beziehungen nach allen Richtungen und glänzende dimensionale Betrachtungen.»

1927 verliess Debye die ETH, da er einen Ruf nach Leipzig annahm. Wiederum half Zangger bei der Suche nach einem Nachfolger. Gleichzeitig verlor die Universität Erwin Schrödinger, der nach Berlin berufen wurde. Um diese Zeit weilte Zangger oftmals in Berlin mit dem Auftrag, dort den gerichtlich-medizinischen Unterricht zu reorganisieren. Am 21. Oktober teilte er Einstein mit ${ }^{60}$ :

«Lieber Einstein! In Zürich ist in der Physik noch gar nichts geschehen. Gestern kam Scherrer zu mir, heute der Dekan der philosophischen Fakultät [II der Universität]. [Walter] Bothe [der dann 1954 den Nobelpreis erhielt] tritt in den Vordergrund, und ich habe auch mit meiner Überzeugung nicht zurückgehalten, nachdem ich ihn mit Präsident Paschen im Reichsphysikalischen Institut besucht habe und einen sehr guten Eindruck von ihm habe. Dagegen ist die theoretische Physik in ganz schlimmer Lage. Heisenberg hat noch nicht abgesagt, aber man rechnet täglich mit seiner Absage, weil er nach Leipzig gehe zu Debye.

So entschuldigen Sie mich, wenn ich nach Besprechungen auch mit andern Kollegen, die mich besucht haben, weil sie immer glauben, ich werde wieder einen Einstein holen, mich an Sie wende. Nun muss Einstein helfen!

Wissen Sie, ob [Paul] Dirac, dessen Name ich Ihnen in Berlin nannte, nach Zürich käme und ob er schon in Deutschland längere Zeit gewesen ist, dass er bald deutsch vortragen könnte? Mit Dirac würde man bald durchdringen. [Sein Vater stammte aus der Schweiz.]

Es scheint, dass Edgar Meyer [Wolfgang] Pauli nicht gern haben möchte. Dann besteht auch die Absicht, man solle für die Universität, die ja nicht so viel auslegen kann, einen glücklichen Griff tun und einen ganz jungen Kollegen herbekommen der nachher in die Linie Einstein, Schrödinger, Debye passen würde.

Ich habe einen Moment an Ihren Mitarbeiter [Leon] Szilard (Ungar) gedacht der doch recht geschickt ist und in der Diskussion sehr wesenhaft anpackte, eingriff, wenn er auch bei einem andern Mal etwas abglitt, der Ideen hat und sehr gut und eindringlich darstellt. Anderseits besteht die Schwierigkeit, Nicht-Habilitierte zu berufen, leider Gottes.

Aber wenn Sie einen empfehlen können, von dem man einen so allgemein guten Eindruck hat, wird die Fakultät zu gewinnen sein und Edgar Meyer lässt sich in dieser Situation wohl auch überzeugen. Man fragte mich auch nach einem jungen Physiker [Richard] Tolman in Pasadena. Können Sie raten?

Kramers und andere Kopenhagener werden wohl kaum weggehen.

Was denken Sie für die technische Hochschule, falls Heisenberg nicht kommt? Von den jüngeren Wienern? und von [Gregor] Wentzel in Leipzig? der ja, falls Heisenberg zu Debye geht, offenbar von Leipzig wegginge. Er hat ja gute Ideen, würde auch der Technik nicht so fern stehen ${ }^{61}$. Ich habe ihn nur einmal gesehen, als beweglichen Menschen. Sein physikalisches Können kann ich zu wenig beurteilen ...

Ich möchte nur ganz vertraulich Ihnen vorschlagen, was jetzt möglich ist; Scherrer, Bothe als experimentelle und gut theoretische gebildete Physiker. Ein Professor für technische Physik, Messmethoden: [Franz] Tank oder [Walter] Dällenbach ${ }^{62}$ »

60 Zangger an Einstein, 21. Oktober 1927, ETH.

61 G. Wentzel wandte sich u.a. auch konkreteren Problemen zu, wie z.B. der Theorie der Rückdiffusion; deshalb die Bemerkung, dass er der Technik näher stehe als andere Theoretiker.

62 F. Tank, der Privatdozent an der Universität Zürich war, wurde 1922 Professor für Physik, später auch für Hochfrequenztechnik. Walter Dällenbach, Privatdozent an der ETH, hatte als Elektroingenieurstudent Einsteins sämtliche Vorlesungen gehört und bei H. Weyl doktoriert, galt aber als Person etwas schwierig. 
Einstein muss wohl geantwortet haben, da in einem neuen Brief an Einstein vom 7. November $1927^{63}$ Zangger kommentierte:

«Mit Tank haben Sie offenbar Recht; ich habe eine Vorlesung von ihm gehört, die war durchaus nicht klar; ich fand auch keine irgendwie originelle ideen oder Darstellungsweise, und das war ein Gebiet, das ich beurteilen konnte.

Ich habe Scherrer nun noch einmal sehr deutlich, fast hart geschrieben; da auch er diese gute Meinung von Bothe hatte, wäre es nicht richtig, $z$ u warten bis Bothe irgend eine andere Stelle annimmt und dann sich zu ärgern. Auswahl von Menschen jener Art, die so gut zu uns passen würden, ist nicht nur in Berlin nicht gross!

An Brillouin und [Louis] de Broglie haben wir auch gedacht; [der Physikochemiker an der Universität, Victor] Henri behauptet, sie kämen nicht. [...] Wegen [Pascual] Jordan müsste man sich noch genauer erkundigen.»

Später im Winter 1927/28 klagte Zangger bei Einstein ${ }^{64}$ :

«Ich glaube nicht, dass der [ETH-]Schulratspräsident Rohn die Physik ausbaut, Debye ist empört. Ich habe dies auch gefürchtet, so weit ich die Menschen erfahren habe. Ich weiss nicht, was ich Bothe sagen soll.»

Schliesslich berief die ETH Wolfgang Pauli auf den Lehrstuhl für theoretische Physik, den er vom Sommer 1928 bis zu seinem Tod 1956 inne hatte. Scherrer blieb jedoch der einzige Experimentalphysiker, und für die technische Physik wurde Tank berufen. An der Universität wurde Wentzel der Nachfolger von Schrödinger. Damit waren alle Ordinariate in Physik an den beiden Hochschulen bis nach Zanggers Emeritierung 1941 besetzt.

So hat Zangger während drei Dekaden selbstlos, auch wenn er sich dabei gelegentlich unbeliebt machte, zum hohen Ruf der Physik an beiden Zürcher Hochschulen beigetragen. Er bewies ständig grossen Weitblick und gutes Urteilsvermögen, denn alle seine vorgeschlagenen Physiker waren schon oder wurden später in ihrem Fach sehr prominent.

\section{Dank}

Gina Zangger, die den Nachlass ihres Vaters im elterlichen Haus bewahrt, danke ich herzlichst für ihre Gastfreundschaft und Hilfe bei der Suche nach dem Quellenmaterial. Ich danke auch den Albert Einstein Archiven an der Hebrew University in Jerusalem für die Erlaubnis, Briefe Einsteins zu zitieren. Robert Schulmann vom Einstein Papers Project hat mir nicht nur mit vielen Auskünften geholfen; ganz besonders wertvoll und meine Arbeit sehr erleichternd waren die hauptsächlich von ihm stammenden vielen Erklärungen und Hinweise auf Dokumente oder Begebenheiten in den Fussnoten in Band 5 der Collected Papers of Albert Einstein. Beat Glaus, dem Leiter der Wissenschaftshistorischen Sammlungen der ETH, sowie Felix Lehner bin ich ebenfalls für ihre Mithilfe zu Dank verpflichtet.

63 Zangger an Einstein, 7. November 1927, Collected Papers 13.

64 Zangger an Einstein, Winter 1927/28, Ebd. 